\title{
Profiles of family pet ownership during the COVID-19 pandemic
}

\author{
Eli D. Halbreich ${ }^{1}$ (D) Megan K. Mueller ${ }^{2,3}$ (])
}

Accepted: 24 November 2021

(c) The Author(s), under exclusive licence to Springer Science+Business Media, LLC, part of Springer Nature 2021

The pandemic caused by SARS-CoV-2 (COVID-19) has affected both people and animals globally (Bowen et al., 2020). Research on COVID-19 with both children and adults has indicated negative psychological impacts on mental health outcomes and stress (Odriozola-González et al., 2020; Rothe et al., 2021; Saddik et al., 2021), and significant variation in mental health outcomes by demographic variables (Pierce et al., 2020).

Pets are an underexplored source of emotional support that may foster resilience within families in times of stress (Bowen et al., 2020; Bussolari et al., 2021; Chadwin, 2017). However, research investigating the mental health benefits of pet ownership prior to COVID-19 has produced mixed outcomes (e.g., Powell et al., 2019) and further research is needed to elucidate the potential benefits of companion animals (Gee \& Mueller, 2019; Wells, 2019). Human-pet relationships have the potential to be amplified-both positively and negatively - during times of crisis. For example, the effects of unemployment and resource scarcity during the pandemic may intersect significantly with pet ownership. The perception that companion animals may reduce loneliness may be driving increased pet acquisition, and some initial data have suggested that adoption rates increased during the pandemic (Morgan et al., 2020). However, relinquishment of pets may be related to economic stress associated with the pandemic, suggesting a need to explore transitions in pet ownership in the context of psychological well-being.

Existing research on pets and COVID-19 suggests that it is imperative to understand sociodemographic profiles of pet ownership during times of increased societal stress, to better

Eli D. Halbreich

Eli.Halbreich@tufts.edu

1 School of Arts and Sciences, Tufts University, 490 Boston Avenue, Medford, MA 02155, USA

2 Cummings School of Veterinary Medicine at Tufts University, North Grafton, MA, USA

3 Tisch College of Civic Life at Tufts University, Medford, MA, USA understand how pets contribute to psychological well-being for diverse families. Therefore, this study aims to extend existing work with convenience samples to explore the role of pets during COVID-19 using a nationally-representative sample of United States families to assess if there are systematic sociodemographic differences between families with and without pets during COVID-19, and explore if these differences are related to acquisition of a pet.

\section{Methods}

\section{Participants and Procedure}

This study used data from the Adolescent Brain Cognitive Development (ABCD) Study®. We analyzed youth data from the ABCD COVID Rapid Response Research Survey, which was administered electronically three times: May 16-22, June 24-27, and August 4-5, 2020. Parent-reported demographic data are from the ABCD Annual Curated Release 3.0, collected between September 1, 2016 and February 15, 2020.

For this analysis, we used a subsample of 8,158 participants who had data for pet ownership in at least one of the three surveys. Youth reported their age (ranging from 10.6 to 14.8 years) and gender. Parents/guardians reported their child's racial/ethnic identities, which were combined into non-mutually exclusive analytic categories of White, Black/ African American, Asian, Indigenous, and Hispanic. See Table 1 for demographic data.

\section{Measures}

\section{Household Income}

Parents/guardians reported their combined family income for the past 12 months on a 10-point scale $(1=$ Less than $\$ 5,000 ; 10=\$ 200,000+)$. 
Table 1 Descriptive statistics/frequencies for demographic/contextual characteristics, stratified by pet ownership status

\begin{tabular}{|c|c|c|c|c|c|c|c|}
\hline & Non-Pet Owners & Pet Owners & Dog Owners & Cat Owners & Other Pet Owners & Pet to No Pet & No Pet to Pet \\
\hline & $n=2,099$ & $n=5,849$ & $n=3,032$ & $n=1,705$ & $n=301$ & $n=106$ & $n=191$ \\
\hline & $M(S D)$ & $M(S D)$ & $M(S D)$ & $M(S D)$ & $M(S D)$ & $M(S D)$ & $M(S D)$ \\
\hline Household Income & $6.93(2.51)$ & $7.87(1.95)$ & $7.88(1.92)$ & $7.64(2.04)$ & $7.62(2.04)$ & $7.21(2.31)$ & $7.14(2.38)$ \\
\hline Sex & $n(\%)$ & $n(\%)$ & $n(\%)$ & $n(\%)$ & $n(\%)$ & $n(\%)$ & $n(\%)$ \\
\hline Female & $1,044(45.2 \%)$ & $2,921(49.9 \%)$ & $1,533(50.6 \%)$ & $896(52.6 \%)$ & $163(51.3 \%)$ & $48(45.3 \%)$ & $105(55.0 \%)$ \\
\hline Male & $1,265(54.8 \%)$ & $2,928(50.1 \%)$ & $1,499(49.4 \%)$ & $809(47.4 \%)$ & $155(48.7 \%)$ & $58(54.7 \%)$ & $86(45.0 \%)$ \\
\hline \multicolumn{8}{|l|}{ Race/Ethnicity } \\
\hline White & $1,426(61.8 \%)$ & $5,077(86.8 \%)$ & $2,640(87.1 \%)$ & $1,543(90.5 \%)$ & $279(87.7 \%)$ & $77(72.6 \%)$ & $135(70.7 \%)$ \\
\hline Black & $713(30.9 \%)$ & $618(10.6 \%)$ & $317(10.5 \%)$ & $137(8.0 \%)$ & $40(12.6 \%)$ & $21(19.8 \%)$ & $46(24.1 \%)$ \\
\hline Asian & $218(9.4 \%)$ & $386(6.6 \%)$ & $200(6.6 \%)$ & $103(6.0 \%)$ & $15(4.7 \%)$ & $9(8.5 \%)$ & $15(7.9 \%)$ \\
\hline Indigenous & $52(2.3 \%)$ & $210(3.6 \%)$ & $121(4.0 \%)$ & $64(3.8 \%)$ & $12(3.8 \%)$ & $3(2.8 \%)$ & $7(3.7 \%)$ \\
\hline Other Race & $187(8.1 \%)$ & $325(5.6 \%)$ & $158(5.2 \%)$ & $70(4.1 \%)$ & $15(4.7 \%)$ & $6(5.7 \%)$ & $17(8.9 \%)$ \\
\hline Hispanic & $501(21.7 \%)$ & $958(16.4 \%)$ & $517(17.1 \%)$ & $238(14.0 \%)$ & $50(15.7 \%)$ & $25(23.6 \%)$ & $51(26.7 \%)$ \\
\hline \multicolumn{8}{|l|}{ Education } \\
\hline HS or less & $379(16.4 \%)$ & $580(9.9 \%)$ & $309(10.2 \%)$ & $179(10.5 \%)$ & $41(12.9 \%)$ & $12(11.3 \%)$ & $23(12.0 \%)$ \\
\hline Some College & $1,326(57.4 \%)$ & $3,463(59.2 \%)$ & $1,801(59.4 \%)$ & $1,088(63.8 \%)$ & $199(62.6 \%)$ & $64(60.4 \%)$ & $114(59.7 \%)$ \\
\hline Graduate degree + & $601(26.0 \%)$ & $1,799(30.8 \%)$ & $919(30.3 \%)$ & $436(25.6 \%)$ & $78(24.5 \%)$ & $30(28.3 \%)$ & $54(28.3 \%)$ \\
\hline \multicolumn{8}{|l|}{ Experienced scarcity } \\
\hline No scarcity & $1,793(77.7 \%)$ & $4,936(84.4 \%)$ & $2,559(84.4 \%)$ & $1,397(81.9 \%)$ & $259(81.4 \%)$ & $88(83.0 \%)$ & $151(79.1 \%)$ \\
\hline Scarcity & $516(22.3 \%)$ & $913(15.6 \%)$ & $473(15.6 \%)$ & $308(18.1 \%)$ & $59(18.6 \%)$ & $18(17.0 \%)$ & $40(20.9 \%)$ \\
\hline \multicolumn{8}{|l|}{ Employment } \\
\hline Not employed & $728(31.5 \%)$ & $1,424(24.3 \%)$ & $719(23.7 \%)$ & $436(25.6 \%)$ & $84(26.4 \%)$ & $31(29.2 \%)$ & $51(26.7 \%)$ \\
\hline Employed & $1,547(67.0 \%)$ & $4,299(73.5 \%)$ & $2,242(73.9 \%)$ & $1,219(71.5 \%)$ & $222(69.8 \%)$ & $74(69.8 \%)$ & $135(70.7 \%)$ \\
\hline
\end{tabular}

Dog, cat, and pet ownership categories are not mutually exclusive

\section{Parent Education}

Parents/guardians reported their highest level of education in 20 categories from never attended/kindergarten through professional/doctoral degree, which was recoded into three categories (see Table 1).

\section{Scarcity}

Parents/guardians were asked to report whether they had experienced specific instances of scarcity in the past year: could not afford: food, telephone service, rent/mortgage, utilities, medical care, dental care; were evicted for not paying rent/mortgage. A dichotomous composite variable was created to indicate if families had experienced at least one of these circumstances.

\section{Employment}

Parents/guardians reported their current employment status in 12 categories, which was recoded into a dichotomous variable (employed, unemployed).

\section{Pet Ownership}

Youth were asked whether they currently have any pets ( $1=$ Yes; $0=$ No), and to specify the type/species of pets. Participants were considered an overall pet owner if they consistently had a pet during the three time points (with similar coding by species for dog, cat, and other pet categories). To assess transitions in pet ownership, participants were categorized as 1) consistent pet owners (had a pet at all time points with data), 2) consistent non-pet owners (no pet at any time point), 3 ) pet to no pet (had a pet at the first and/or second time point but not the second and/or third time point), and 4) no pet to pet (did not have a pet at the first and/or second time point but had a pet at the second and/or third time point).

\section{Data Analysis}

Analyses were performed in SPSS 27.0. Logistic regression was used to assess how sociodemographic variables predicted overall pet ownership, pet ownership by species, and pet ownership consistency status. Prior to analysis, variables were tested for the necessary assumptions for regression 


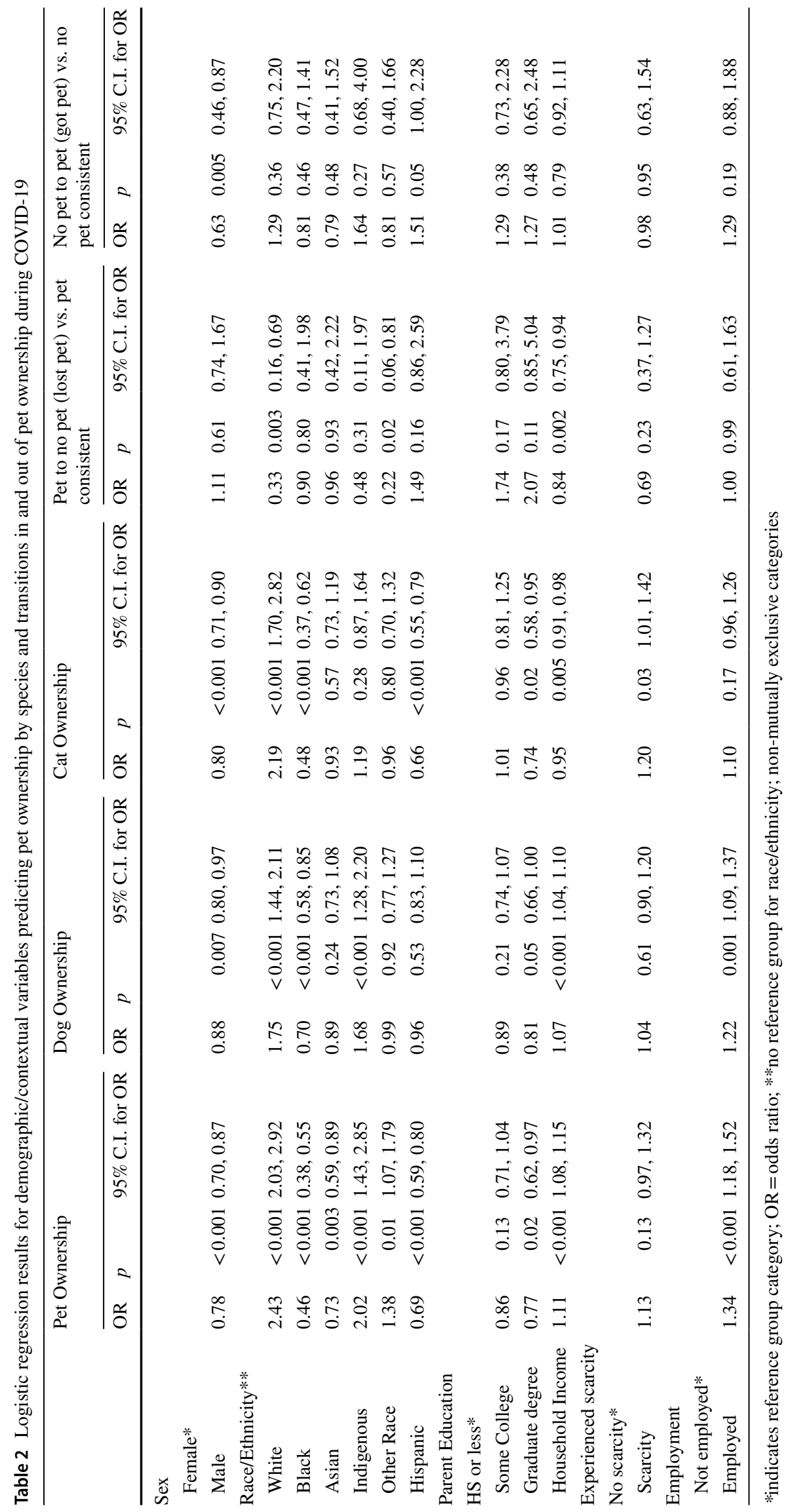


modeling. To interpret significance, we report p-values, odds ratios, and $95 \%$ confidence intervals.

\section{Results}

Regression analyses indicated that odds of pet ownership differed significantly on several sociodemographic variables. Higher odds of pet ownership were associated with identifying as White or Indigenous, and income was positively associated with pet ownership. Being male, Black, Asian, Hispanic, and having a parent with a graduate degree were all associated with lower odds of pet ownership. There were some differential patterns by species for dog and cat owners regarding race/ethnicity, experiences of scarcity, and employment (see Table 2). Participants whose family transitioned out of pet ownership during the pandemic were less likely to be White and more likely to have reported lower household income compared to consistent pet owners. Male participants were more likely to have acquired a pet compared to female participants. Table 2 includes full regression results.

\section{Discussion}

Results indicated that there are significant systematic differences in the experience of pet ownership based on sociodemographic variables. Specifically, the findings related to families transitioning from having a pet to not having a pet during COVID-19 point to potential systemic inequities and economic factors (e.g., access to veterinary care and job loss; Applebaum et al., 2020) driving some relinquishments. These findings are similar to other studies looking at sociodemographic factors in pet ownership from prior to COVID-19 (e.g., Mueller et al., 2021). Additionally, a relatively small number of families changed their pet ownership status, which could indicate the presence of protective factors-such as enhanced unemployment insurance relief-or the importance of pets within the family context.

A limitation of this analysis is that we do not know the reasons why families either lost (through relinquishment, death, or other reasons) or acquired (through a relative or friend, or adoption) pets during COVID-19. This study provides initial evidence that pet ownership is a factor in the lives of many families but there is significant inter-individual variation. Although these findings are within the context of COVID-19, external and internal stressors will continue to affect families going forward, and so the precise role of pets within the family needs further exploration in the context of psychological well-being.
Authors' Contributions Conceptualization: Eli D. Halbreich, Megan K. Mueller; Methodology: Megan K. Mueller; Formal analysis and investigation: Megan K. Mueller; Writing —original draft preparation: Eli D. Halbreich; Writing_review and editing: Megan K. Mueller; Funding acquisition: Megan K. Mueller; Resources: Megan K. Mueller; Supervision: Megan K. Mueller.

Funding The analyses for this study were funded by the HumanAnimal Bond Research Institute (HABRI), grant \#HAB19-001. This manuscript reflects the views of the authors and may not reflect the opinions or views of HABRI.

Data used in the preparation of this article were obtained from the Adolescent Brain Cognitive Development ${ }^{\mathrm{SM}}$ (ABCD) Study (https:// abcdstudy.org), held in the NIMH Data Archive (NDA). This is a multisite, longitudinal study designed to recruit more than 10,000 children age 9-10 and follow them over 10 years into early adulthood. The ABCD Study ${ }^{\circledR}$ is supported by the National Institutes of Health and additional federal partners under award numbers U01DA041048, U01DA050989, U01DA051016, U01DA041022, U01DA051018, U01DA051037, U01DA050987, U01DA041174, U01DA041106, U01DA041117, U01DA041028, U01DA041134, U01DA050988, U01DA051039, U01DA041156, U01DA041025, U01DA041120, U01DA051038, U01DA041148, U01DA041093, U01DA041089, U24DA041123, U24DA041147. A full list of supporters is available at https://abcdstudy.org/federal-partners.html. A listing of participating sites and a complete listing of the study investigators can be found at https://abcdstudy.org/consortium_members/. ABCD consortium investigators designed and implemented the study and/or provided data but did not necessarily participate in the analysis or writing of this report. This manuscript reflects the views of the authors and may not reflect the opinions or views of the NIH or ABCD consortium investigators. Additional support for this work was made possible from supplements to U24DA041123 and U24DA041147, the National Science Foundation (NSF 2028680), and Children and Screens: Institute of Digital Media and Child Development Inc.

The $\mathrm{ABCD}$ data repository grows and changes over time. The $\mathrm{ABCD}$ data used in this report came from the ABCD Annual Curated Release 3.0 (https://doi.org/10.15154/1519007) and the COVID Rapid Response Research (RRR) Survey First data release (https://doi.org/ 10.15154/1520584).

Data Availability The datasets generated during and/or analyzed during the current study are available in the ABCD Annual Curated Release 3.0 (https://doi.org/10.15154/1519007) and the COVID Rapid Response Research (RRR) Survey First data release (https://doi.org/ $10.15154 / 1520584)$.

Code Availability Analyses were performed in SPSS (IBM Corp. Released 2020. IBM SPSS Statistics for Windows, Version 27.0. Armonk, NY: IBM Corp). Analysis code is publicly available via Open Science Framework: https://osf.io/hct46/?view_only=480dd 9ff3a9446d5927545a6a583d785.

\section{Declarations}

Conflicts of Interest/Competing Interests The authors have no conflicts of interest to declare that are relevant to the content of this article.

Ethics Approval This study was approved by each local ethics committee of the relevant institutions, including but not limited to the University of California, San Diego (ABCD Study Coordinating Center). The procedures used in this study adhere to the tenets of the Declaration of Helsinki. 
Consent to Participate All adult participants (parents/guardians) provided written informed consent, and all youth provided written assent along with permission from a parent/guardian.

Consent for Publication Participants signed informed consent regarding publishing their data as part of their participation in the ABCD Study.

\section{References}

Applebaum, J. W., Tomlinson, C. A., Matijczak, A., McDonald, S. E., \& Zsembik, B. A. (2020). The concerns, difficulties, and stressors of caring for pets during COVID-19: Results from a large survey of U.S. pet owners. Animals, 10, 1882. https://doi.org/10.3390/ ani10101882

Bowen, J., García, E., Darder, P., Argüelles, J., \& Fatjó, J. (2020). The effects of the Spanish COVID-19 lockdown on people, their pets, and the human-animal bond. Journal of Veterinary Behavior, 40, 75-91. https://doi.org/10.1016/j.jveb.2020.05.013

Bussolari, C., Currin-McCulloch, J., Packman, W., Kogan, L., \& Erdman, P. (2021). "I couldn't have asked for a better quarantine partner!": Experiences with companion dogs during Covid-19. Animals, 11(2), 330. https://doi.org/10.3390/ani11020330

Chadwin, R. (2017). Evacuation of pets during disasters: A public health intervention to increase resilience. American Journal of Public Health, 107(9), 1413-1417. https://doi.org/10.2105/AJPH. 2017.303877

Gee, N. R., \& Mueller, M. K. (2019). A systematic review of research on pet ownership and animal interactions among older adults. Anthrozoös, 32(2), 183-207. https://doi.org/10.1080/08927936. 2019.1569903

Morgan, L., Protopopova, A., Birkler, R. I. D., Itin-Shwartz, B., Sutton, G. A., Gamliel, A., Yakobson, B., \& Raz, T. (2020). Human-dog relationships during the COVID-19 pandemic: Booming dog adoption during social isolation. Humanities and Social Sciences Communications, 7, 155. https://doi.org/10.1057/ s41599-020-00649-x

Mueller, M. K., King, E. K., Callina, K., Dowling-Guyer, S., \& McCobb, E. (2021). Demographic and contextual factors as moderators of the relationship between pet ownership and health. Health Psychology and Behavioral Medicine, 9(1), 701-723. https://doi.org/10.1080/21642850.2021.1963254

Odriozola-González, P., Planchuelo-Gómez, Á., Irurtia, M. J., \& de Luis-García, R. (2020). Psychological effects of the COVID-19 outbreak and lockdown among students and workers of a Spanish university. Psychiatry Research, 290, 113108. https://doi.org/10. 1016/j.psychres.2020.113108

Pierce, M., Hope, H., Ford, T., Hatch, S., Hotopf, M., John, A., Kontopantelis, E., Webb, R., Wessely, S., McManus, S., \& Abel, K. M. (2020). Mental health before and during the COVID-19 pandemic: A longitudinal probability sample survey of the UK population. The Lancet Psychiatry, 7(10), 883-892. https://doi.org/10.1016/ s2215-0366(20)30308-4

Powell, L., Edwards, K. M., McGreevy, P., Bauman, A., Podberscek, A., Neilly, B., Sherrington, C., \& Stamatakis, E. (2019). Companion dog acquisition and mental well-being: A community-based three-arm controlled study. BMC Public Health, 19, 1428. https:// doi.org/10.1186/s12889-019-7770-5

Rothe, J., Buse, J., Uhlmann, A., Bluschke, A., \& Roessner, V. (2021). Changes in emotions and worries during the Covid-19 pandemic: An online-survey with children and adults with and without mental health conditions. Child and Adolescent Psychiatry and Mental Health, 15, 11. https://doi.org/10.1186/s13034-021-00363-9

Saddik, B., Hussein, A., Albanna, A., Elbarazi, I., Al-Shujairi, A., Temsah, M.-H., Saheb Sharif-Askari, F., Stip, E., Hamid, Q., \& Halwani, R. (2021). The psychological impact of the COVID-19 pandemic on adults and children in the United Arab Emirates: A nationwide cross-sectional study. BMC Psychiatry, 21, 224. https://doi.org/10.1186/s12888-021-03213-2

Wells, D. L. (2019). The state of research on human-animal relations: Implications for human health. Anthrozoös, 32(2), 169-181. https://doi.org/10.1080/08927936.2019.1569902

Publisher's note Springer Nature remains neutral with regard to jurisdictional claims in published maps and institutional affiliations. 